\title{
Research on Cloud Computer and Library Management Challenges
}

\author{
Tao Ren ${ }^{1}$ \\ ${ }^{1}$ Xi'an External Affairs Institute, Xi'an, Shanxi, 710077 \\ hunter2011@foxmail.com
}

Keywords: Cloud Computer, Library Management, Challenges

\begin{abstract}
Associated with cloud computing, network technology and service has been applied to the library. Cloud computing will bring enormous benefits to library and will also bring challenges to library management. Management challenges faced by libraries include: substitutability issue, standards, data security and privacy issues, intellectual property issues. To address these challenges, library science theorists should study: basic theoretical issues of cloud computing, cloud computing applications in the feasibility of the library, a library of cloud computing policies, standards and protocols, cloud-based library management system, the cloud calculation cases.
\end{abstract}

\section{Introduction}

In recent years, the rapid rise of cloud computing in computer network technology has become a new data processing technology, not only bring certain convenience to people's lives, but also has been applied to various fields. In the management of the library, the application of cloud computing greatly improves the management of digital libraries, information technology, of course, but it also brings some challenges to the management of the library. In this paper, the library computing challenges bring by cloud is started from the analysis, and then to explore and put forward some measures, in order to provide some reference for future management of the library.

Library as an important reserve of knowledge of the place of its management model along with the continuous development of science and technology, gradually to digital network management model developed from the traditional manual management mode, coupled with recent years, cloud computing this new data processing appear the way, but also makes network management library become an inevitable trend, but also accelerated the pace of its development, cloud computing has brought great convenience to the library at the same time, we should also see their faces behind challenges, as well as measures to address these challenges in China.

\section{Cloud Computing and Libraries}

Cloud computing concept of time appeared not long, but the generation technology background and driving force is very complex cloud computing, the researchers mixed background and vision, so the concept of cloud computing many and messy. Matrix has summed up the 20 definition of cloud computing, but still far from being able to cover the existing definition. Andy Isherwood, vice president of Hewlett-Packard more bluntly: Many people join the cloud trend, but I have not heard two people say the same thing. Conceptual confusion is one reason the library hostage Supremacy computing research. Library people, should be appropriate to discuss with the concept of cloud computing to maintain a certain distance, but will focus on the theory concerns the application of cloud computing.

In terms of look angle of the classification of cloud computing application library, you can see the current level, mainly platform and software level applications. In fact, the greatest challenge to the library cloud is the issue from the infrastructure level. IT paradigm shift and power infrastructure NicholasCarr cloud computing will be the first result of comparing: Today, we are in a new era of upheaval a century ago it occurred in the generation of everything taking place in the information processing. The computer system by the private individual companies built and run by the public grid serious that the Internet, being squeezed out of the data service center provided treatment plant. Computing becomes a utility, like electric utilities, the new utility computing is 
reaching far more than most companies in their own system can achieve economies of scale. Cloud computing compared with electricity and water supply, and indeed can help us better understand the meaning and value of cloud computing. In a power infrastructure in developed societies, ordinary businesses rely on self-generating power is unthinkable. After the cloud computing infrastructure, the library may be able to own all the hardware and software outsourcing to a cloud, like electricity, like paying on time. Imagine, if this pattern was established, it will bring big changes to IT management library.

\section{Challenges To The Library Management Brought by Cloud Computing}

Cloud computing has now been widely applied to various fields, researchers have related it to conduct a comprehensive study, it was found that cloud computing applications there are some problems in the work of library management:

Standard question: At the moment, cloud computing, does not have a unified, standardized criteria, the criteria used now either standard Microsoft products, or is related industry organizations to develop standards, so in practice because of standards problems caused by more and more obvious, if not a unified standard, and so instead of freely transferable between different vendors is difficult to achieve interoperability between different cloud can not be achieved. In addition, the technology, cloud computing is also an urgent need for a common standard to support. While in the library industry, this standard is more demanding, interactive interface program first requires standardization, and secondly, you also need to support the standardization of data and so on.

Substitutability problem: During library cloud computing applications, the library can be freely selected IT vendors, rather than rely entirely on the same service provider, and at the time for different suppliers can also choose different services project, which is in the cloud with the appropriate on the basis of substitutability to complete. If you do not have this feature, then the library will depend on cloud computing, which is bound suppliers, the consequences of such a result is unthinkable.

Data security and privacy issues: The management of the library, the data security and confidentiality has always been an extremely important content, whether it is the reader's information data or data library of books, in the event of lost or leaked, it will be a serious loss, and even cause some of the consequences that can not imagine. Therefore, the challenge of the cloud to bring the library, how to ensure data security and confidentiality of information has also become one of the most important challenges. For cloud computing, all the data libraries are stored in the cloud is not very safe, but in fact is about the same on the local hard disk and on the nature and, therefore, need something here on the policy level to constraints, such as national policy, cloud computing and other enterprise management policy.

Intellectual property issues: Generally speaking, the library in the cloud service after purchase, will own content in the form of data to the cloud, then the cloud computing business management of these relevant content, while users still have to Library All rights of these contents, can make the appropriate changes, even delete files. That can be delivered to the cloud content management, and cloud computing companies can not make any operation, but in the current cloud computing companies, many enterprises in the users get the content, the content will be relevant integration and use, here issues related to intellectual property rights, it is because of the existence of this phenomenon, which has become a challenge to cloud computing brings to library management.

\section{The Method to Deal with the Challenges of Library Management}

Strengthening basic theoretical research cloud computing. Cloud computing as a new information processing technology, in theory and method of cloud computing technology for continuous in-depth study and exploration is a very important work. At this stage, the basic theory of cloud computing mainly includes several aspects, namely the origin of cloud computing, concepts, basic theory, types and characteristics, etc., although these studies did not involve cloud computing in Library Management practical application, however, the library management through 
cloud computing theoretical basis of in-depth study and understanding of the problem, it can make in the future work of library management can better respond to the challenges posed by cloud computing.

Strengthen the feasibility study in the library of cloud computing applications. China currently has been adhering to the traditional concept of the library is "owned", and cloud computing service concept is the data stored in the cloud, which makes a certain degree of conflict between the two, while, allowing managers to Library Can not accept. Therefore, the feasibility study library management application cloud computing has become a problem in library management needed to solve. First, the actual situation of the library, will be reasonably applied to cloud computing related areas, such as automated management systems, storage and sharing of data, and so on, so that the benefits of cloud computing research can bring to the library, improving Books level museum management.

Strengthen research policy management standard library cloud computing. In the cloud management standards and policies, we must intensify research to develop appropriate management charter and standards as soon as possible, so as to ensure cloud computing to meet the needs of library management. Although it has introduced a number of cloud computing corresponding application protocols and standards, but some are not suitable for use in the library community, therefore, in the development of the library community cloud management standards and policies of the agreement, it shall be based on the Library Management the actual situation, the standard cloud computing research, thereby to form a suitable library community science industry standards and protocols. In addition, cloud providers should strengthen research, particularly its main cloud computing providers sharing, openness and normative. Only in this way, in the management of the library in order to give full play to the role of cloud computing, so security interests but also to better protect the libraries and data.

Strengthen cloud computing services library management system. With increasing levels of information technology, cloud computing in the library management application has become a big trend, then a question to consider is, the application of cloud computing once in library management, it is necessary to original The management system changes, and even the original may also make the entire management system changes, so that when using cloud computing for library management, library management, what impact it will bring, it is necessary to taken into account. Therefore, in cloud-based library management services, and to strengthen its management system becomes very important.

Strengthen the case studies of cloud computing. Chinese current library management application cloud computing is not a lot of cases, but there are some representative cases, such as the Amazon API, Google Books API, etc., associated staff can conduct research and analysis of these cases, then combined their actual situation to determine the advantages and disadvantages of these cases, so as to continuously improve and innovate, to find a method for their own cloud-based library management program, which is a good deal with the challenges brought about by cloud computing .

With the growing popularity of cloud computing and development, cloud computing has been applied in various fields, the library community is no exception, so the cloud-based library management will face a multitude of opportunities and challenges in this Under the circumstances, it must be brought to challenge the cloud of a comprehensive analysis of library management and library combined with our own actual situation, to find a suitable own management plan, build a scientific management mode, so as to respond to the challenges of cloud computing brings library management, the role of cloud computing will play out in the real library management and achieve effective management library.

\section{Conclusion}

In a word, as a center to carry out research and education of universities, the library occupies an important position in the development process of colleges and universities. With the development of computer technology and network technology, cloud computing has been widely used in the work of library management, resulting in library management faced with enormous opportunities and 
challenges, in this case, in order to achieve effective management of university libraries, they should conduct a comprehensive and systematic analysis for challenge brings to library management by cloud computing. Combined with the management of university library, we should establish university library management model and make full use of cloud computing challenge brought to the library management, so as to maximize the role of the library.

\section{References}

[1] Burrows M. The Chubby Lock Service for Loosely-Coupled Distributed Systems.2006.

[2] Daniel J Abadi. Data Management in the Cloud: Limitations and Opportunities.2009.

[3] Clark C, Fraser, K, Hand S, etc. A Live Migration of Virtual Machines.2005.

[4] Sims K.IBM. Introduces Ready-To-Use Cloud Computing Collaboration Services Get Clients Started with Cloud Computing.2007. 